Original Article

\title{
Properties of muscle nodules recognized by manual physical therapy practitioners
}

\author{
KaOru Isogai, RPT, MS ${ }^{1 *}$, Shogo Okamoto, $\mathrm{PhD}^{2)}$, Yoji Yamada, PhD²) \\ 1) Department of Physical Therapy, Faculty of Health and Medical Sciences, Tokoha University: 1230 \\ Miyakoda-cho, Kita-ku, Hamamatsu-shi, Shizuoka 431-2102, Japan \\ 2) Department of Mechanical Systems Engineering, Graduated School of Engineering, Nagoya University, \\ Japan
}

\begin{abstract}
Purpose] The purpose of this study was to describe the properties of muscle nodules (kinkoketsu) recognized by manual physical therapy practitioners. [Participants and Methods] A total of one hundred and thirtythree physical therapists, occupational therapists, judo therapists, and acupuncturists participated in this study. The shape, size, direction, depth, and hardness of muscle nodules, often treated in the buttocks, were investigated through the completion of a questionnaire. [Results] A total of 124 answer sheets were completed; 112 of these described the shape of muscle nodules as ellipsoidal. Of these 112 sheets, 97 effective sheets were analyzed. The results showed that the mean long axis length, short axis length, and thickness of the muscle nodules were $30.9 \mathrm{~mm}$, $16.2 \mathrm{~mm}$, and $9.3 \mathrm{~mm}$, respectively. The most common responses on the long axis direction, depth, and hardness of the muscle nodules were the craniocaudal orientation, the second shallowest layer of 5 divisions, and the eraser level, respectively. [Conclusion] The typical muscle nodule found in the buttocks by manual physical therapy practitioners is roughly the shape of a large almond.

Key words: Manual physical therapy, Muscle nodule, Palpation
\end{abstract}

(This article was submitted Nov. 30, 2020, and was accepted Jan. 21, 2021)

\section{INTRODUCTION}

In the field of manual physical therapy in Japan, areas of local muscle hardness that are associated with muscle pain are called muscle nodules ("kinkoketsu" in Japanese). A variety of terms were used for such areas of hardness in overseas reports from the early 19 th century to the early 20 th century ${ }^{1)}$. These included thickenings, nodular tumors, muscle callouses, fibrositis, and myogeloses ${ }^{1}$. However, the relationship between these areas of hardness and muscle nodules has not been verified. At the end of the 20th century, the concept of trigger points was proposed by Travell et al. ${ }^{2}$, and this has become a central topic of research in the field of muscle pain. Initially, trigger points were reported to be tender spots in taut bands of the muscle that, when stimulated, caused patients to exhibit local twitch responses, referred pain, or the jump sign ${ }^{2}$. These reactions suggested that the trigger point was a spot in the muscle distinct from muscle nodules. Later, "nodules in a taut band" were added to the diagnostic criteria of trigger points ${ }^{3)}$ and are now seen as palpable nodules. This new criterion may have caused confusion between trigger points and muscle nodules in Japan.

When muscle nodules are deemed to be the cause of muscle pain or other symptoms, many manual physical therapy practitioners (MPTPs) in Japan try to improve the symptoms with techniques such as pressing the muscle nodules with their fingers ${ }^{4}$. To accomplish this, it is essential to locate the muscle nodules that cause the symptoms. As palpation is generally used to search for muscle nodules, tactile sensations are vital for their identification. However, no criteria for assessing muscle nodules have been established, and the field has been unable to formally define a muscle nodule. While muscle

*Corresponding author. Kaoru Isogai (E-mail: kisogai@hm.tokoha-u.ac.jp)

(C2021 The Society of Physical Therapy Science. Published by IPEC Inc.

(c) (i) $\odot$ This is an open-access article distributed under the terms of the Creative Commons Attribution Non-Commercial No DerivaCC BY NC ND tives (by-nc-nd) License. (CC-BY-NC-ND 4.0: https://creativecommons.org/licenses/by-nc-nd/4.0/) 
nodules are routinely treated in the field of manual physical therapy in Japan, there is little shared awareness of their pathology and physical properties. Therefore, MPTPs identify muscle nodules based on their own study and clinical experience. During this process, they also recognize the shape, size, hardness, and other attributes of muscle nodules. However, MPTPs have no means of assessing the accuracy of the muscle nodule properties they recognize. Thus, there is a need to demonstrate the typical properties of muscle nodules to provide MPTPs with a standard. Moreover, the results of our study can be used as a guide when creating the muscle nodule models or dummies for education ${ }^{5)}$. Since muscle nodules are recognized through clinical practice, we need to investigate the MPTPs' opinions about muscle nodules. Thus, we opted to use a questionnaire survey. The purpose of this study is to reveal the size, hardness, and other properties of muscle nodules recognized by MPTPs and also identify the relationships among these attributes.

\section{PARTICIPANTS AND METHODS}

Participants were sourced from the list of those who attended seminars held by the Society for Surface Anatomy in Aichi, Osaka, Kochi, and Kumamoto prefectures. Amongst these, individuals who confirmed that they regularly treated muscle nodules in clinical practice were asked to respond to a questionnaire. Responses were obtained from 133 practitioners (age $29.9 \pm 7.2$ years; clinical experience $6.1 \pm 5.7$ years), comprising 111 physical therapists, 14 occupational therapists, 3 judo therapists, 2 certified as both judo therapists and acupuncturists, and 3 certified as both masseurs and acupuncturists. The content of the study was explained to all the participants, and their consent was obtained in writing. This study was carried out with the approval of the Tokoha University Research Ethics Committee (approval No. 2014-015H).

The questionnaire was structured around 5 items relating to muscle nodules; these were shape, size, direction, depth, and hardness. In addition, to help the participants recall specific muscle nodules, they were asked to only consider muscle nodules found in the buttocks. Muscle nodules are often found in areas such as the neck, shoulders, and low back. However, because the buttocks have large muscles and are supported by a flat bone, they were considered the most suitable area of study as their recollection was unlikely to be affected by complex anatomical structures.

To limit the area of the hypothetical muscle nodule in the buttocks, the questionnaire contained a photograph of the buttocks with a point marked between the anterior superior iliac spine (ASIS) and the posterior superior iliac spine (PSIS). The participants were asked to recall muscle nodules they had often treated near this mark and to draw an outline of how a nodule would look when viewed perpendicularly to the body surface on the questionnaire. They were asked to depict the muscle nodule in its realistic shape and size. They were also asked to draw the nodule considering its direction relative to the craniocaudal direction of the body.

As we believed it would be difficult for the participants to accurately recall and draw the 3-dimensional shape of a muscle nodule, they were only asked to provide the thickness of the nodule in millimeters. To obtain the depth of the muscle nodules, a diagram of a cross-section of the subcutaneous tissue and muscle between the ASIS and PSIS was included in the questionnaire. In addition, we scanned this site on a man with a standard physique using ultrasound, and based on the results, the thicknesses of the subcutaneous tissue and muscle in the diagram were set to $10 \mathrm{~mm}$ and $40 \mathrm{~mm}$, respectively. The thickness of the muscle on the diagram was then divided into 5 equal parts of $8 \mathrm{~mm}$ each (from shallow to deep: Depth 1, Depth 2, Depth 3, Depth 4, and Depth 5), and the participants were asked to select the depth where the muscle nodule they recalled was located. As for hardness, due to the difficulty of providing a numerical answer, the participants were asked to give the name of an object with a hardness similar to that of the muscle nodule they recalled.

On the outlines of the muscle nodules drawn on the questionnaire, we located the 2 points that were furthest apart and defined the long axis as the line between these points, and the short axis was the line orthogonal to this. The long and short axes on each figure were measured with a ruler to calculate their mean and standard deviation. For thickness, the mean and standard deviation were calculated from the figures given in the questionnaire. Using the long axis, short axis, and thickness values of the muscle nodules, we calculated the Mahalanobis distances of samples (a scale measuring the distance from the centroid of data points when there are correlations between multiple variables in multivariate data), and excluded all responses that were outside the $95 \%$ of samples closest to the center of mass as outlier values. Figures in which the long axis was 1.5 times or longer than the short axis were classified as having the long axis either in the craniocaudal or the mediolateral direction of the buttocks. Muscle nodule hardness was divided into 4 levels based on the similarity of the hardness of the objects described in the responses. Factor analysis was applied to the 5 items of long axis length, short axis length, thickness, depth, and hardness of the muscle nodules. Factors were selected based on whether the eigenvalues were $\geq 1$ (Kaiser-Guttman criterion $\left.^{6}\right)$, and promax rotation was applied to calculate the loadings. $\mathrm{R}$ was used to calculate the Mahalanobis distances and for the factor analysis (mahalanobis function, factanal function, ver. 3.6.1).

\section{RESULTS}

Of the 133 questionnaires, 9 were excluded from the analysis because the muscle nodule diagram was not depicted in a single line, and thus the shape and size could not be determined. The outlines of muscle nodules on the remaining 124 questionnaires were classified by shape based on their similarity. There were 112 round nodules $(90.3 \%)$ that were circular or elliptical, 4 rectangular nodules (3.0\%), 3 spindle-shaped nodules $(2.3 \%), 2$ linear nodules (1.5\%), 2 U-shaped nodules 
(1.5\%), and 1 triangular nodule $(0.8 \%)$. Therefore, we determined that the typical muscle nodule shape recognized by MPTPs was round. As a result, the 112 questionnaires with round outlines were chosen as the candidates for further analysis. Two of these questionnaires did not have responses for thickness, and an additional 2 had thickness values that were larger than the thickness of the muscle. These 4 questionnaires were excluded from the analysis. From the remaining 108 questionnaires, 6 were further excluded due to the long axis, short axis, or thickness values being outliers. From the remaining 102 questionnaires, 4 that did not name any object resembling the hardness of the muscle nodule and 1 that named several objects of different hardness levels were excluded from the analysis. As a result, the final analysis set comprised 97 questionnaires. Of these 97 questionnaires, 80 were completed by physical therapists, 11 by occupational therapists, 2 by judo therapists, 1 by a doubly qualified judo therapist and acupuncturist, and 3 by doubly qualified masseurs and acupuncturists (age, $29.7 \pm$ 7.1 years; clinical experience, $5.9 \pm 5.5$ years).

The mean and standard deviation of the long and short axes in the diagrams of muscle nodules in the 97 questionnaires were $30.9 \pm 15.5 \mathrm{~mm}$ and $16.2 \pm 8.4 \mathrm{~mm}$, respectively, with median values of $28 \mathrm{~mm}$ and $14 \mathrm{~mm}$, respectively (Fig. 1a, b). The mean muscle nodule thickness was $9.3 \pm 6.7 \mathrm{~mm}$, and the median was $6.5 \mathrm{~mm}$ (Fig. 1c). In 74 of the 97 questionnaires (76.3\%), the long axis was at least 1.5 times longer than the short axis. In 65 of these 74 questionnaires (87.8\%), the long axis was close to having a craniocaudal orientation, and in $9(12.2 \%)$ it was close to a mediolateral orientation.

Depth 2 (8-16 mm from the surface of the muscle) was the most common nodule depth out of the 38 responses (Fig. 1d). Based on the responses on nodule hardness, gummy candy, eraser, cork, and stone were used as reference objects for the 4 levels of hardness. All the objects described in the responses were subsequently classified into these 4 levels based on their similarity to the hardness of the reference object. For example, jello and tapioca pearl were classified as gummy level, bouncy ball, and tennis ball were eraser level, tire and baseball were cork level, and peanut and plum seed were stone level. The most common hardness level was the eraser level (Fig. 1e).

As the eigenvalues of factors 1 to 3 in the factor analysis were 1.91, 1.14, and 0.96, we adopted 2 factors. Table 1 shows the factor loadings of the 5 variables. Factor 1 had high loadings for the long axis and short axis, with only a slight loading for thickness. Factor 2 was related to depth. Because their uniqueness values were close to 1, the thickness and hardness were hardly explained by 2 factors. In the correlation matrix shown in Table 2, the long axis was strongly correlated with the short axis but only weakly correlated with depth. Further, the short axis was weakly correlated with thickness, and thickness was weakly correlated with depth. In contrast, muscle nodule hardness was independent of the other characteristics.
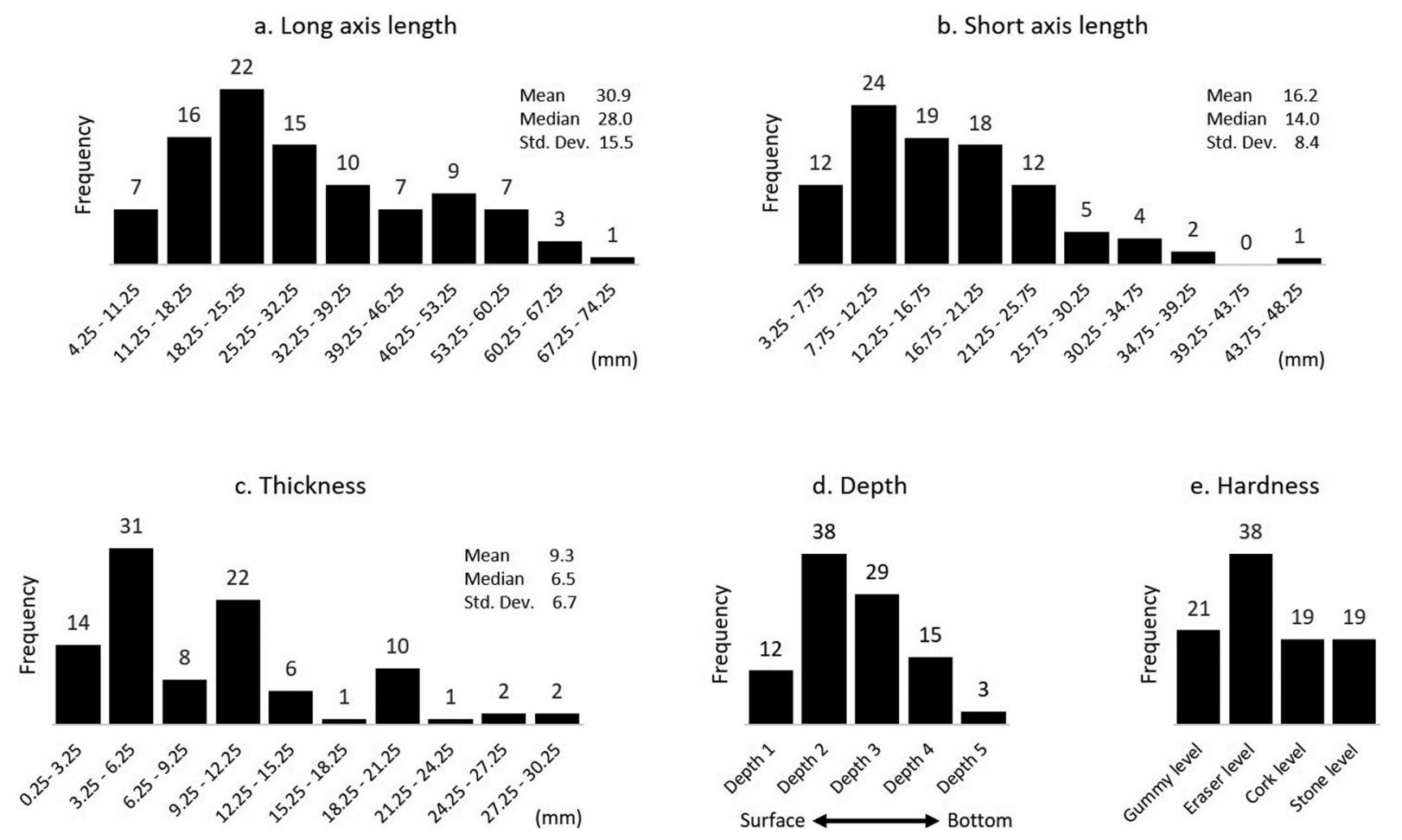

Fig. 1. Histograms of muscle nodule size, depth, and hardness responses. 


\section{DISCUSSION}

The outlines of muscle nodules obtained in this study indicate that the nodules recognized by many MPTPs have an elliptical shape when viewed perpendicularly to the body surface. While only numerical responses were obtained for muscle nodule thickness, it seems unlikely that the elliptical lesions recognized by the MPTPs extended in the direction of thickness to form an elliptic cylinder. Similar to other tumors, this shape most likely represents nodules with rounded edges rather than those with corners. Therefore, it seems reasonable that the most recognized 3-dimensional shape of the nodule was an ellipsoid. Applying the results of this study to the ellipsoid shape indicates that the typical muscle nodule in the buttocks recognized by MPTPs has the shape of a large almond. In addition, the long axis of this almond-shaped body is in the craniocaudal direction, located slightly shallower than the middle of the muscle, and is about as hard as an eraser (Fig. 2).

We used mean and mode values to describe the properties of a typical muscle nodule. However, many of the responses differed from this typical example. For example, the standard deviations of the long axis, short axis, and thickness were $15.5 \mathrm{~mm}, 8.4 \mathrm{~mm}$, and $6.7 \mathrm{~mm}$, respectively, and the mean values were $30.9 \mathrm{~mm}, 16.2 \mathrm{~mm}$, and $9.3 \mathrm{~mm}$, respectively. This shows that MPTPs recognized a large range of muscle nodule sizes. In addition, the depth responses ranged from Depth 1 to Depth 5, and hardness responses were almost evenly distributed across all 4 levels. The results of the factor analysis suggest that the long and short axes of muscle nodules are correlated when viewed perpendicularly to the body surface. It is reasonable that MPTPs recognize nodules that are elliptically shaped with lengths that are about twice their width, even if the sizes differ. However, thickness, which is another indicator of size, is only weakly correlated with the lengths of the long and short

Table 1. Factor loadings

\begin{tabular}{lccc}
\hline & Factor 1 & Factor 2 & Uniqueness \\
\hline Long axis length & 0.77 & & 0.40 \\
Short axis length & 0.87 & & 0.26 \\
Thickness & 0.28 & 0.20 & 0.86 \\
Depth & & 1.00 & 0.00 \\
Hardness & 0.11 & -0.16 & 0.97 \\
\hline
\end{tabular}

Table 2. Correlation coefficients between research variables

\begin{tabular}{lccccc}
\hline & 1 & 2 & 3 & 4 & 5 \\
\hline 1. Long axis length & 1 & & & & \\
2. Short axis length & 0.660 & 1 & & & \\
3. Thickness & 0.232 & 0.271 & 1 & 1 & \\
4. Depth & 0.178 & 0.088 & 0.248 & -0.138 & 1 \\
5. Hardness & 0.104 & 0.040 & 0.118 & & \\
\hline
\end{tabular}

a

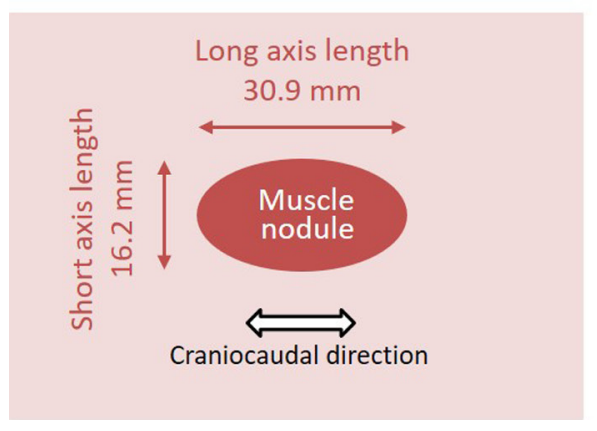

b

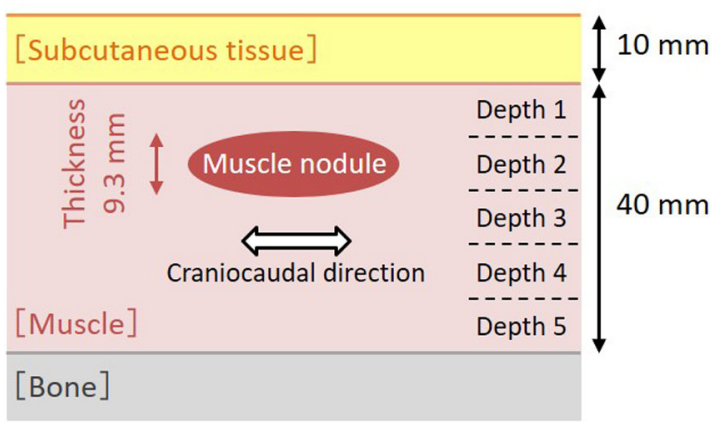

Fig. 2. Diagram of the typical muscle nodule recognized by MPTPs. a: View of the muscle nodule perpendicular to the surface of the body. b: View of the muscle nodule parallel to the surface of the body. 
axes. This indicates that smaller muscle nodules are recognized as more spherical and larger muscle nodules are recognized as having a flatter shape. Alternatively, because the muscle nodules in this study were assumed to be in a relatively flat area of the buttocks, which is a large muscle, these anatomical characteristics may have influenced the results. Furthermore, muscle nodule thickness was weakly related to depth. This is a reasonable result as thicker nodules are less likely located near the surface of the muscle.

The reason why muscle nodule hardness did not correlate at all with the other properties could be due to differences in the pressure used by MPTPs upon palpation. Although the pathogenesis of muscle nodules is unclear, the difficulty of finding them during palpation suggests that they are unlikely to have extremely distinct boundaries with the surrounding tissue. Moreover, if muscle nodules are harder near their centers and show normal muscle hardness near the borders, MPTPs who depicted larger muscle nodules would be expected to recognize softer lesions; however, this tendency was not observed. The stress-strain curve of muscles has been reported to exhibit strong nonlinearity ${ }^{7}$. According to this report, muscle tissue was very soft when pressed very slightly. A gradual increase in pressure of the thumb caused the hardness to increase rapidly. When pressure was continued beyond this point, the degree of hardness prevented further collapse. This suggests that muscle nodules palpated with weak pressure were of the gummy-candy-level hardness, while nodules palpated with strong pressure were of stone-level hardness. Therefore, the recognition by MPTPs of muscle nodule hardness depends on the amount of pressure used during palpation, and the pressure can be different according to the career of practitioners ${ }^{8)}$. This could explain the distribution of hardness levels across the 4 levels.

The relationship between muscle nodules and trigger points remains unclear. Recently, trigger points and taut bands have been visually identified using ultrasound and nuclear magnetic resonance in an attempt to quantify their shape, size and hardness ${ }^{9-14}$. These reports examined the trapezius and low back region and found that trigger points are elliptically shaped, but with an area of only $0.16-0.57 \mathrm{~cm}^{2,9-11)}$. Further, hardness values of the taut bands have been reported at 9.0 $\mathrm{kPa}, 36.3 \mathrm{kPa}$, and $11.5 \mathrm{kPa}$, almost 1.5 to 2 times that of normal muscle ${ }^{12-14)}$. These values indicate that taut bands are much softer than gummy candy. However, because these size and hardness values were extracted from images when the tissue was not subjected to pressure, we cannot conclude that they are completely different from the objects in the present study, as our values were perceived indirectly through subcutaneous tissue. Therefore, while it is possible that the muscle nodules recognized by MPTPs include trigger points or taut bands, or at the very least their characteristics, ultrasound and nuclear magnetic resonance data suggest that they are not the same as those reported by MPTPs. If in the future criteria for muscle nodules can be established and muscle nodules can be identified in images, progress could be made on verifying their relationship with trigger points and taut bands.

We could not find any methods or devices for objectively quantifying the properties of muscle nodules recognized by MPTPs. As a result, we opted to use a questionnaire survey, despite its methodological limitations. One of these limitations is that it is impossible to verify the accuracy of the participant's depiction of the shape and size of the muscle nodules they are recalling. When classifying the shapes of the muscle nodule outlines, the similarity between the hand-drawn outline and regular shapes was assessed by the authors. In addition, we did not obtain specific responses about the shape of the nodules in the direction of thickness. Finally, we did not verify the agreement with the participants in the classification of the objects' hardness provided in the responses. Therefore, we were unable to eliminate the subjectivity of the authors in the assessment that muscle nodules are almond shaped and have the hardness of an eraser. Furthermore, it is possible that having the participants depict muscle nodules as single-line drawings and having them name objects with the homogeneous hardness falsely guided them toward seeing muscle nodules as having a simple structure. Therefore, participants might have reported muscle nodules as having uniform hardness when, in fact, the nodule shapes they palpate are more complex.

Muscle nodules probably have various pathologies, and differences in the severity of muscle pain or stage of the disease could lead to different attributes. Hence, MPTPs with different job qualifications may experience different muscle nodules. However, we could not verify this because most of the study participants were physical therapists or occupational therapists.

The recognition of muscle nodule properties may also vary depending on the participant's educational background and may change as they gain more clinical experience. Therefore, the conclusions we can make about typical muscle nodule properties based on mean and mode values are limited. Yet, with the study of muscle nodules at a stalemate, advancements in manual physical therapy also seem to be at a stop. We hope that this study will stimulate reflection on our perceptions of muscle nodules, thus leading to increased discussion among MPTPs. The outcomes for the field due to these renewed properties about muscle nodules will lead to the development of their diagnostic criteria.

In this study, we investigated the properties of muscle nodules recognized by MPTPs. The results indicate that a typical muscle nodule in the buttocks is about the shape and size of a large almond. Moreover, the long axis of a typical muscle nodule is oriented in the craniocaudal direction, is slightly shallower than the middle of the muscle, and is about as hard as an eraser.

\section{Presentation at a conference}

Part of this study was presented at the 49th Congress of the Japanese Society of Physical Therapy.

\section{Funding}

This work was supported in part by JSPS KAKENHI Grant No. JP24500520. 


\section{Conflict of interest}

There authors have no conflicts of interest to declare regarding this study.

\section{REFERENCES}

1) Shah JP, Thaker N, Heimur J, et al.: Myofascial trigger points then and now: a historical and scientific perspective. PM R, 2015, 7: 746-761. [Medline] [CrossRef]

2) Travell JD, Simons DG: Myofascial pain and dysfunction: the trigger point manual. Baltimore: Williams \& Wilkins, 1983, pp 59-63.

3) Simons DG, Travell JG, Simons LS: Travell \& Simons' myofascial pain and dysfunction: The trigger point manual. Vol. 1. Upper half of body, 2nd ed. Baltimore: Lippincott Williams \& Wilkins, 1999, pp 116-122.

4) Kogo H, Kurosawa K: Seeking the cause of myofascial pain syndrome by identifying which manual therapy is effective against muscle tenderness and stiffness J Phys Ther Sci, 2010, 22: 173-176. [CrossRef]

5) Isogai K, Okamoto S, Noda A, et al.: Training on muscle palpation using artificial muscle nodule models, Haptic Interaction: Science. Eng Des, 2016, 432: 335-340.

6) Yeomans KA, Golder PA: The Guttman-Kaiser criterion as a predictor of the number of common factors. J R Stat Soc D, 1982, 31: 221-229.

7) Isogai K, Okamoto S, Yamada Y, et al.: Skin-fat-muscle urethane model for palpation for muscle disorders. Proceedings of IEEE/SICE International Symposium on System Integration, 2015, pp 960-964.

8) Okuyama T, Yokoyama S, Tanahashi Y, et al.: Measurement of palpation motion using prostate examination simulator and motion capture system. Mech Eng J, 2016, 3: 15-00539. [CrossRef]

9) Sikdar S, Shah JP, Gebreab T, et al.: Novel applications of ultrasound technology to visualize and characterize myofascial trigger points and surrounding soft tissue. Arch Phys Med Rehabil, 2009, 90: 1829-1838. [Medline] [CrossRef]

10) Ballyns JJ, Shah JP, Hammond J, et al.: Objective sonographic measures for characterizing myofascial trigger points associated with cervical pain. J Ultrasound Med, 2011, 30: 1331-1340. [Medline] [CrossRef]

11) Cojocaru MC, Cojocaru IM, Voiculescu VM, et al.: Trigger points-ultrasound and thermal findings. J Med Life, 2015, 8: 315-318. [Medline]

12) Chen Q, Bensamoun S, Basford JR, et al.: Identification and quantification of myofascial taut bands with magnetic resonance elastography. Arch Phys Med Rehabil, 2007, 88: 1658-1661. [Medline] [CrossRef]

13) Chen Q, Basford J, An KN: Ability of magnetic resonance elastography to assess taut bands. Clin Biomech (Bristol, Avon), 2008, 23: 623-629. [Medline] [CrossRef]

14) Chen Q, Wang HJ, Gay RE, et al.: Quantification of myofascial taut bands. Arch Phys Med Rehabil, 2016, 97: 67-73. [Medline] [CrossRef] 